\title{
The Effects of Toxoplasmosis and Malaria Coinfection on Malaria Parasite Density and Hematological Parameters in Children (0-6 Years) in the Nkolbisson Health District, Cameroon
}

\author{
Samuel Nambile Cumber ${ }^{1,2, ~}$, Kameni Nguekam Yvonne-Estelle ${ }^{2}$, Shalom Jaila ${ }^{2}$ \\ ${ }^{1}$ Discipline of Public Health Medicine, Department of Nursing \& Public Health, College of Health Sciences, University of Kwazulu-Natal, \\ Durban, South Africa \\ ${ }^{2}$ Under Privilege Children and Women Assistance (UPCAWA-SWEDEN), Bamenda, Cameroon
}

Email address:

samuelcumber@yahoo.com (S. N. Cumber)

${ }^{*}$ Corresponding author

\section{To cite this article:}

Samuel Nambile Cumber, Kameni Nguekam Yvonne-Estelle, Shalom Jaila. The Effects of Toxoplasmosis and Malaria Coinfection on Malaria Parasite Density and Hematological Parameters in Children (0-6 Years) in the Nkolbisson Health District, Cameroon. Journal of Family Medicine and Health Care. Vol. 2, No. 4, 2016, pp. 81-88. doi: 10.11648/j.jfmhc.20160204.19

Received: September 18, 2016; Accepted: October 15, 2016; Published: November 23, 2016

\begin{abstract}
Toxoplasma gondii and Plasmodium species are both endemic apicomplexan parasites that have been incriminated in the cause of febrile illnesses in children in the sub-Saharan regions of Africa. Moreover these parasites have some common routes of transmission, common receptors for pathogenicity and both effect or of some hematological parameters. Despites this, little is known about the prevalence of toxoplasmosis and malaria co-infection in Cameroon and their effects on hematological parameters and malaria parasite density. Venous blood was collected from 315 febrile children in the Nkolbisson Health District found in Yaoundé Cameroon. For each participant: RDT for Toxoplasma gondii, Toxoplasma gondii IgG avidity test, thick film microscopy and full blood count was performed. The prevalence of toxoplasmosis was $40 \%$, malaria $42.8 \%$ and toxoplasmosis and malaria co-infection 20\%. The age group 0-5 years was identified as risk group for both infections and Nkol-Atem had the highest prevalence of both infections. Toxoplasmosis and malaria of co-infection led to a slight increase in RBCs, WBCs, and platelets counts in our study population. This could therefore be suggestive of a mechanism between the two parasites that may improve the physiology of blood cells production. However the presence of a co-infection did not show any influence on the malaria parasite density. This study provides valuable information on the prevalence of malaria and toxoplasmosis co-infection in Cameroonian children where data is almost unavailable. This study thus indicates a need to enforce control and preventive measures against these infections in Cameroonian children.
\end{abstract}

Keywords: Toxoplasmosis, Malaria, Coinfection, Children, Cameroon

\section{Background}

Apicomplexa form a huge family of parasites that cause many different illnesses in humans and animals, and which includes Plasmodium, the parasite that causes malaria and Toxoplasma gondii, the agent that causes toxoplasmosis. [1]

Toxoplasmosis is becoming a global health hazard as it infects $30-50 \%$ of the world human population. [2] Clinically, the life-long presence of the parasite in tissues of a majority of infected individuals is usually considered asymptomatic. However, a number of studies show that this 'asymptomatic infection' may also lead to development of other human pathologies. [3] Apart from toxoplasmosis in immunocompromised individuals, congenital toxoplasmosis is the most serious manifestation of infection and $85 \%$ of live infants with congenital infection appear normal at birth but may develop the disease in a later stage of life. [1-3]

Malaria, a parasitic disease spread by the bite of a mosquito, results in 300 million to 500 million clinical cases and causes more than 1 million deaths yearly. Mostly it is young children under the age of five in sub- Saharan Africa who are affected, dying at the rate of nearly 3,000 every day. [2] Those children who escape death are not untouched by the disease. Malaria also hinders the development of those 
who survive. In sub-Saharan Africa, the disease is responsible for $30 \%$ to $50 \%$ of all outpatient visits to clinics and up to 50 per cent of hospital admissions. [4]

Apart from being endemic in the sub Saharan areas these pathogens have also been incriminated for the cause of febrile illnesses in children. In this line, malarial infections are well known to cause changes in blood cell counts. Hematological changes in the course of a malaria infection, such as anemia, thrombocytopenia and leukocytosis or leucopoenia are well recognized. [5] The hematological aspects equally have some relationship with toxoplasmosis, in most acute toxoplasma infection; the symptoms may be associated with fever, headache, muscle pain, anemia, thrombocytopenia and sometimes lung complications, which are somewhat similar to the symptoms of malaria. $[5,6]$ Therefore the dual presence of these two parasites would be expected to have a more negative effect on the disease severity due to their effects on the hematological parameters. [6]

Furthermore these diseases can be acquired through congenital transmission amongst other similar routes such as poor environmental sanitation, overcrowding, poverty, which could promote co-infection in children. [7] several approaches to target malaria but little is known about the interaction of these related members of the apicomplexan and their effects on the disease severity. Furthermore, few studies have been performed in some African countries including Cameroon where information on the seroprevalence of $\mathrm{T}$. gondii among children is unavailable. $[6,7]$

This cross-sectional study was therefore designed to investigate the seroprevalence of toxoplasmosis and malaria in children, the prevalence of malaria and toxoplasmosis coinfection in children with the aim of understanding their fundamental single and collective effects on hematological parameters in a rural area of the central region of Cameroon. Toxoplasma gondii and Plasmodium species are both endemic apicomplexan parasites that have been incriminated in the cause of febrile illnesses in children in the sub-Saharan regions of Africa. Moreover these parasites have some common routes of transmission, common receptors for pathogenicity and both effector of some hematological parameters. Despites this, little is known about the prevalence of Toxoplasmosis and malaria co-infection and their effects on hematological parameters and malaria parasite density. Hence this study aims at determining the individual prevalence of malaria and toxoplasmosis as well as evaluating the effects of toxoplasmosis and malaria coinfection on malaria parasitemia and some hematological parameters among children (0-16) in the Nkolbisson Health District of Cameroon

This study will be beneficiary because besides providing a better assessment of the rate and burden of these infections in children, the effects of malaria and toxoplasmosis coinfection on the malaria parasitemia and some hematological parameters will be evaluated and the results obtained will help in clinical management of these infections. This study will also improve the knowledge on any adverse effect as a result of the interaction of both parasites.

\section{Methodologies}

\subsection{Study Location}

This research was carried out at the Nkolbisson Health District Yaoundé, Cameroon. The Catholic Health Center Oyom-Abang located in the Nkolbisson Health District, receives many patients from various locations in Nkolbisson. Moreover, healthcare cost is very affordable there by attracting a great number of patients, which provided a good sample size for this study. This Health center is about 15 minutes drive from the Biotechnology Center where samples were stored in the refrigerator and freezer for analysis.

Targeted population: Our target population was Children $0-16$ years. All children who presented with symptoms of a febrile illness and whose guardians concerned to the study were included this study recruited 315 participants.

\subsection{Study Design}

A hospital based cross sectional designed.

\subsection{Study Period}

The study was adopted beginning from the $18^{\text {th }}$ of May to the $18^{\text {th }}$ of June 2015 .

\subsection{Study Sample}

However, for the purpose of this research project, archival samples were used.

\subsection{Data Collection Tool}

A well-structured questionnaire was used for the collection of demographic and clinical data from the participants; venous blood was collected in an EDTA tube. The presence of toxoplasma gondii was ascertained by the use of a colloidal Gold chromatographic cassette (TOX IgG/M rapid test by CTK Biotech-USA). The purpose of this rapid test is to screen for the presence of toxoplasma antibodies. If IgM is identified, proceed with the avidity test to differentiate acute from chronic infection

Principle of the test: The On-site Toxo IgG/IgM Rapid Test is a lateral flow chromatographic immunoassay. The test strip consists of:

1) a burgundy colored conjugate pad containing recombinant $\mathrm{T}$. gondiiantigens conjugated with colloid gold (T. gondii conjugates) and rabbit IgG-gold conjugates.

2) a nitrocellulose membrane strip containing two test bands ( $\mathrm{T} 1$ and $\mathrm{T} 2$ bands) and a control band ( $\mathrm{C}$ band). The $\mathrm{T} 1$ band is pre-coated with monoclonal anti-human IgM for detection of

IgM anti-T. gondii, T2 band is pre-coated with reagents for detection of IgG anti-T. gondii, and the $\mathrm{C}$ band is pre-coated with goat anti rabbit IgG.

When an adequate volume of test specimen is applied into the sample pad of the strip, the specimen migrates by capillary action across the strip. IgM anti-T. gondii if present in the specimen will bind to the $\mathrm{T}$. gondii conjugates. The immunocomplex is then captured on the membrane by the pre-coated anti-human IgM antibody, forming a burgundy colored $\mathrm{T} 1$ band, indicating a $\mathrm{T}$. gondii $\mathrm{IgM}$ positive test 
result. IgG anti-T. gondii if present in the specimen will bind to the T. gondii conjugates. The immunocomplex is then captured by the pre-coated reagents on the membrane, forming a burgundy colored $\mathrm{T} 2$ band, indicating a $\mathrm{T}$. gondii IgG positive test result.

Absence of any $\mathrm{T}$ bands (T1 and T2) suggests a negative result. The test contains an internal control ( $\mathrm{C}$ band) which should exhibit a burgundy colored band of the immunocomplex of goat anti rabbit $\mathrm{IgG} / \mathrm{rabbit} \mathrm{IgG}$-gold conjugate regardless of the color development on any of the $\mathrm{T}$ bands. Otherwise, the test result is invalid and the specimen must be retested with another

Principle of the Test IgM avidity test: Microtiter strip wells coated with Toxoplasma antigen are incubated with diluted serum specimen (dual pipetting). After washing one well is incubated with avidity reagent and the corresponding well with washing buffer. In this step the low avidity antibodies are removed from the antigens whereas the high avidity ones are still bound to the specific antigens. Anti-human IgG labeled with peroxidase is added. The immune complex is visualized with TMB to give a blue reaction product. Stop solution is added to stop the reaction and changing the color of the reaction product into yellow. Absorbance at $450 \mathrm{~nm}$ is read using an ELISA micro well plate reader. Avidity $(\%)>$ 40 implies Toxoplasmosis antibody with high avidity showing past infection, avidity (\%) $\leq 40$ implies toxoplasmosis antibody with low avidity acute or recent infection. [1]

Thick film was prepared using $10 \mathrm{uL}$ of whole blood after drying; the thick blood film was stain with Giemsa stain for 10-15 minutes Thick blood film was examined using the $100 \mathrm{X}$ object for blood stage malaria parasitemia was calculated

Complete blood count was done on the EDTA collected vernous blood samples by the use of the URIT (3200) 3pack fully automated hematology analyser.

\subsection{Data Analysis}

Data was collected using a case report form and entered into a computer database created using Microsoft Excel 2010. Data analysis was implemented using Graph Pad Prism version 6 and the Results obtained are presented on graphs and tables.

\section{Results}

Demographic and clinical characteristics of the study participants

In this study we recruited a total of 315 children with from various parts of Nkolbisson. Males made up 168 (53.5\%) of the study population while females accounted for 147 $(46.7 \%)$ of the total population. The modal age group was 0 5 years which accounted for $180(57.1 \%)$ of the participants, seconded by the age group 6-10 years $86(27.3 \%)$ and the least was recorded by the age group 11-16 49 (15.6\%). With respect to residence $25.4 \%$ of our study participants came from Oyom-Abang, 17.4\% from Camp Sonel, 11.4\% from Nkolbisson, $5.7 \%$ from NkolAtem, 3.5\% from Cite Vert while $36.8 \%$ were from other locations (Table 1 ).
Chief complains cited by/for our study participants included: fever 315 (100\%), abdominal pain 125 (39.7\%), headache $78(24.8 \%)$, vomiting $65(20.6 \%)$ and cough 64 (20.3\%). Other complains included loss of appetite, common cold, diarrhea and very few children complained of joint pains, sore throats, convulsions and respiratory difficulty (Table 2).

Table 1. Demographic characteristic of study participants.

\begin{tabular}{ll}
\hline Characteristics & $\mathrm{N}(\%)$ \\
\hline Gender no. (\%) & \\
Male & $168(53.5)$ \\
Female & $147(46.7)$ \\
Age group no. (\%) & \\
0-5 years & $180(57.1)$ \\
6-10 years & $86(27.3)$ \\
11-15 years & $49(15.6)$ \\
Residence no. (\%) & \\
Nkolbesson & $36(11.4)$ \\
Oyom-Abang & $80(25.4)$ \\
Camp Sonel & $54(17.4)$ \\
Cite Vert & $11(3.5)$ \\
Nkol Atem & $18(5.7)$ \\
Other & $116(36.8)$ \\
\hline
\end{tabular}

Table 2. Clinical characteristic of study participants.

\begin{tabular}{ll}
\hline Characteristics & $\mathrm{N}(\%)$ \\
\hline Chief complaints cited by parents/guardian no. (\%) & \\
Fever & $315 / 315(100)$ \\
Fatigue & $26 / 315(8.3)$ \\
Convulsion & $2 / 315(0.6)$ \\
Sore throat & $6 / 315(2.0)$ \\
Respiratory difficulty & $2 / 315(0.6)$ \\
Diarrhea & $33 / 315(10.5)$ \\
Joint pain & $6 / 315(1.9)$ \\
Headache & $78 / 315(24.8)$ \\
Cough & $64 / 315(20.3)$ \\
Common cold & $27 / 315(8.6)$ \\
Vomiting & $65 / 315(20.6)$ \\
Loss of appetite & $27 / 315(8.6)$ \\
Abdominal pain & $125 / 315(39.7)$ \\
Hematology parameters no/total no. $(\%)$ & \\
Anemia (Hemoglobin $<11 \mathrm{~g} / \mathrm{dL})$ & $144 / 249(57.8)$ \\
Mild anemia $(\mathrm{Hgb} 10-10.9 \mathrm{mg} / \mathrm{dl})$ & $31 / 249(12.4)$ \\
Moderate anemia $(7-9.9 \mathrm{mg} / \mathrm{dl})$ & $52 / 249(20.9)$ \\
Severe anemia $(<7 \mathrm{mg} / \mathrm{dl})$ & $22 / 249(8.8)$ \\
Leukopenia (total WBC $<5 \mathrm{X} \mathrm{10} / \mathrm{L})$ & $56 / 249(22.5)$ \\
Leukocytosis (total WBC $>12 \mathrm{X} \mathrm{10} / \mathrm{L})$ & $37 / 249(14.9)$ \\
\hline
\end{tabular}

Prevalence of toxoplasmosis and malaria coinfection

This study enrolled 316 participants who were tested for the presence of the malaria parasite and toxoplasma gondii. 63 children tested positive for both toxoplasmosis and malaria giving an overall prevalence of $20 \%$ for toxoplasmosis and malaria co-infection.

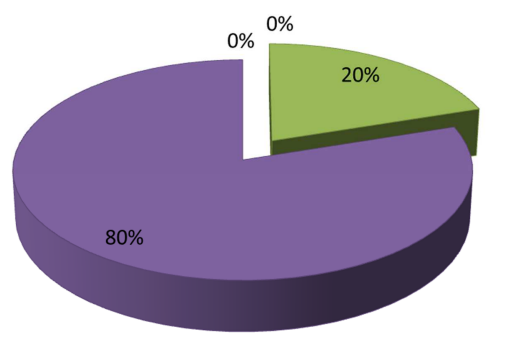

$\square$ toxo and malaria coinfection

$\square$ negative

Figure 1. Prevalence of toxoplasmosis and malaria co-infection amongst children. 


\section{Prevalence of toxoplasmosis and malaria}

Out of 315 participants, 135 were positive for malaria, and 123 were positive for toxoplasmosis with 117 positive for toxoplasma $\operatorname{IgG}$ and 10 positive for toxoplasma $\operatorname{IgM}$ and 4 positive for both toxoplasma IgG and IgM. Therefore giving the prevalence of $42.8 \%$ for malaria, $37 \%$ for toxoplasmosis $\mathrm{IgG}$ and $3.0 \%$ for toxoplasmosis IgM.

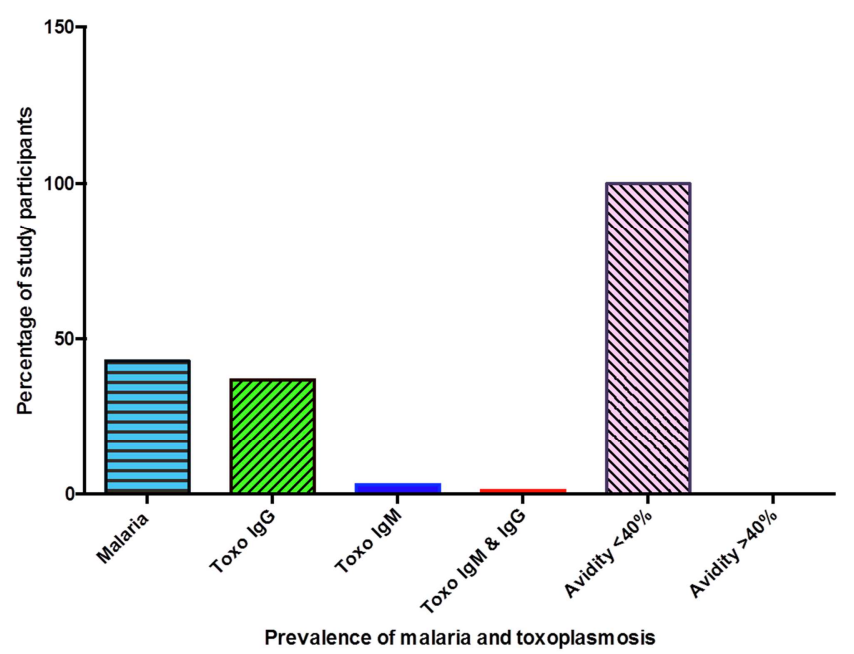

Figure 2. Prevalence of toxoplasmosis and malaria amongst children.

Distributions of toxoplasmosis and malaria according to residence of the participants

Five quarters in the Nkolbisson health district were sampled that is: Nkolbessong, Oyom-Abang, Camp-sonel, cite-vert, nkol-Atem and others. The highest prevalence of toxoplasmosis and malaria were accounted in Nkol-Atem that is $9 / 18(50 \%)$ and $16 / 18(89 \%)$ respectively. The other quarters had distributions as follows for toxoplasmosis and malaria respectively, Nkolbisson recorded 14/36 (39\%); 21/36 (58\%), Camp-Sonel recorded 27/54 (50\%); 38/54 (70\%), Cite-Vert 3/11 (27\%); 5/11 (45\%), Oyom-Abong $35 / 80$ (44\%); 38/80 (48\%) and others 39/116 (34\%); 57/116 $(49 \%)$.

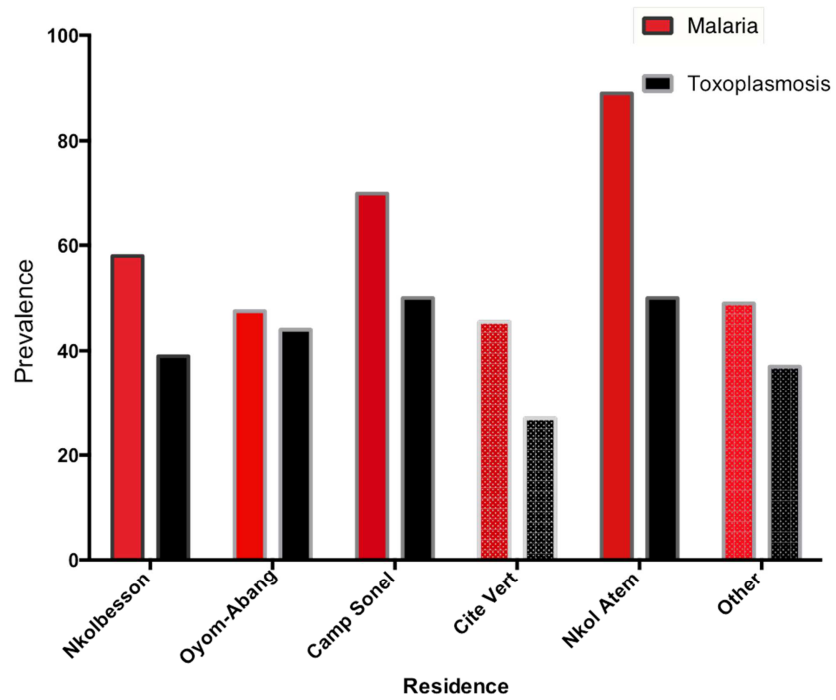

Prevalence of malaria and toxoplasmosis stratified by residence

Figure 3. Prevalence of malaria and toxoplasmosis in children stratified by residence.
Prevalence of malaria and toxoplasmosis according to age groups

The age group of $0-5$ years accounted for the highest prevalence of toxoplasmosis $51(40 \%)$ while the age groups of 6-10 and 11-16 recorded $41(32 \%)$ and 35 (26\%) respectively. The three age groups recorded decreasing prevalence for malaria that is $67(50 \%)$ in the age group $0-5$ years, $40(30 \%)$ in the age group 5-10yrs and $28(20 \%)$ in the age group 11-16.

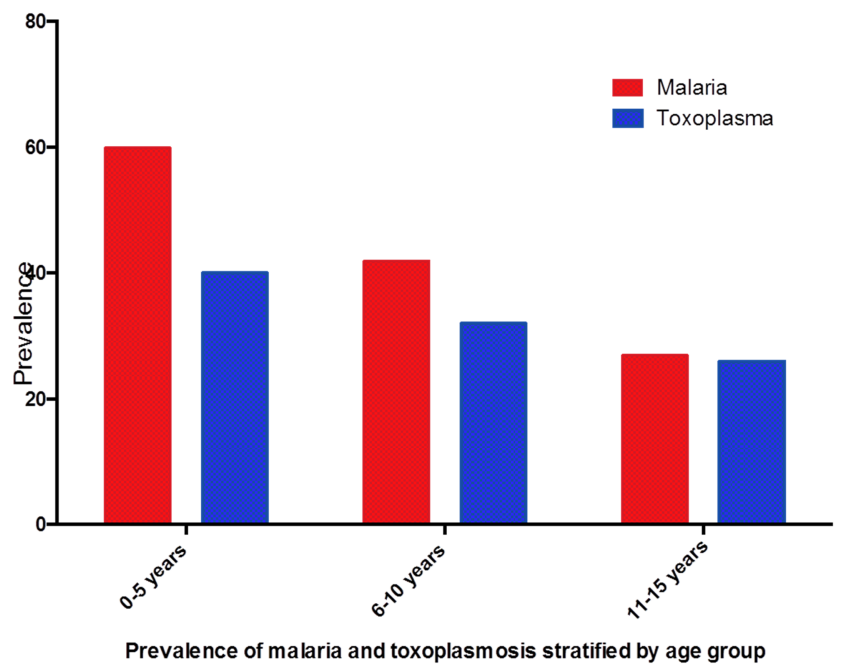

Figure 4. Prevalence of malaria and toxoplasmosis stratisfied by age group.

\section{Prevalence of anemia stratified by age group}

The hemoglobin concentrations were used to classify anemia $(\mathrm{Hgb}<11 \mathrm{~g} / \mathrm{dl})$ as mild $(\mathrm{Hgb} 10-10.9 \mathrm{~g} / \mathrm{dl})$, moderate $(7-9.9 \mathrm{~g} / \mathrm{dl})$ and severe $(<7 \mathrm{~g} / \mathrm{dl})$. Children between $0-5$ years recorded the highest prevalence for mild, moderate and severe anemia respectively $(68 \% ; 69 \% ; 64 \%)$, followed by those between the ages of 6-10 years who recorded a prevalence of $26 \% ; 25 \% ; 27 \%$ respectively and the least in children within the age group of 11-16 years who had a prevalence of $6 \% ; 6 \% ; 9 \%$ respectively for mild, moderate and severe anemia.

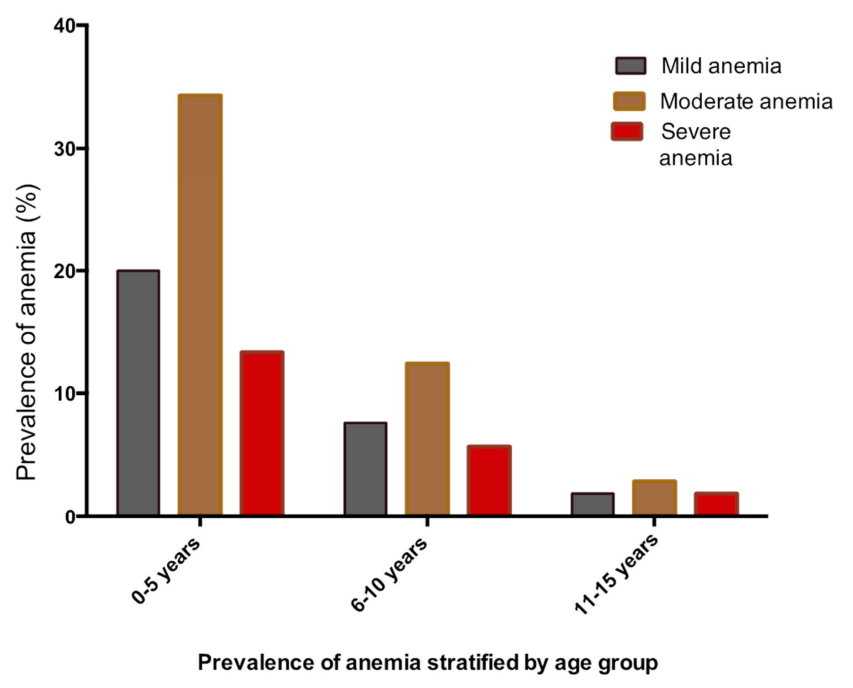

Figure 5. Prevalence of anemia stratified by age group.

Hemoglobin concentrations stratified by infection status The Mann-Whitney test was used to compare the various 
infection status and their effects on hemoglobin. For comparison between those who had malaria only and toxoplasmosis only there was a statistically significant difference in the hemoglobin concentration $(\mathrm{P}=0.0374)$ with the malaria positive group having a lower hemoglobin concentration. There was also a statically significant difference in the hemoglobin concentration in the malaria positive group and those who were negative for both infections $(\mathrm{P}=0.0320)$. There was no statistically significant difference in the hemoglobin concentration in: those who had malaria only and those who had both malaria $(\mathrm{P}=0.0682)$ : those who had toxoplasmosis only and those who had both toxoplasmosis and malaria $(\mathrm{P}=0.6014)$ : those who had toxoplasmosis only and those who had none of the infections $(\mathrm{P}=0.0509)$ : those who had both infections and those who had none of the infections $(\mathrm{P}=0.9280)$.

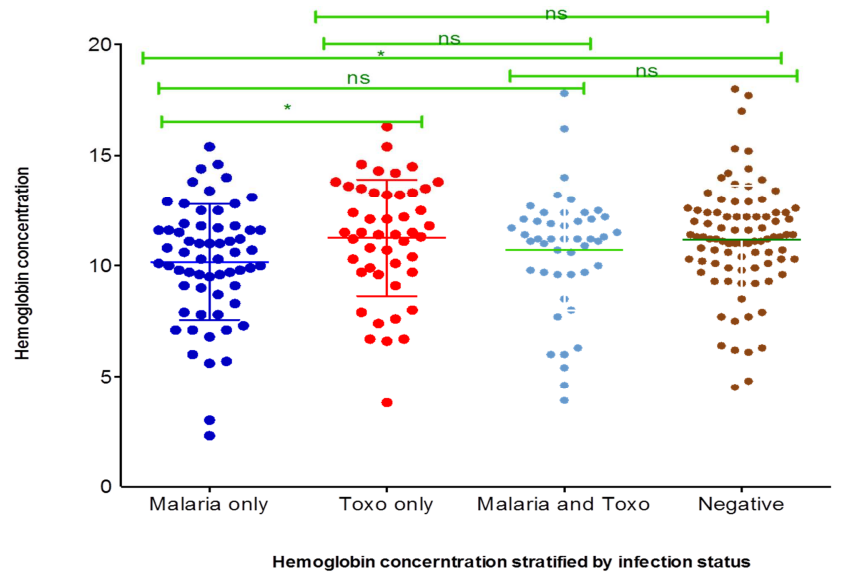

Figure 6. Hemoglobin concentration stratified by infection status.

Parasitemia stratified by infection status

There was no statistical significance difference in the parasite density in those who had malaria only and those who were co-infected with malaria and toxoplasmosis $(\mathrm{P}=0.1241)$.

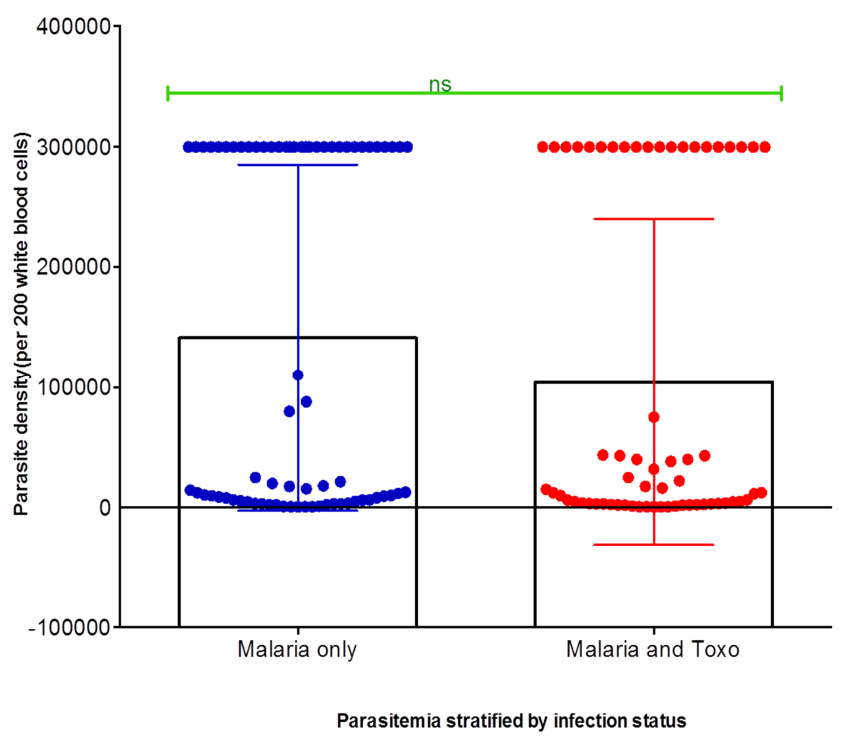

Figure 7. Parasitemia stratified by infection status.

White blood cell count stratified by infection status

There was no statistical significance difference in the total white blood count when the various groups were compared: malaria only and toxoplasmosis only $\mathrm{P}=0.820$, malaria only and malaria/toxoplasmosis co-infection $\mathrm{P}=0.864$, malaria only and those with no infection $\mathrm{P}=0.970$, toxoplasmosis only and malaria and toxoplasmosis co-infection $\mathrm{P}=0.701$ and malaria and toxoplasmosis co-infection and those negative for both infections $\mathrm{P}=0.740$. However the mean white blood cell counts for each the group was different.

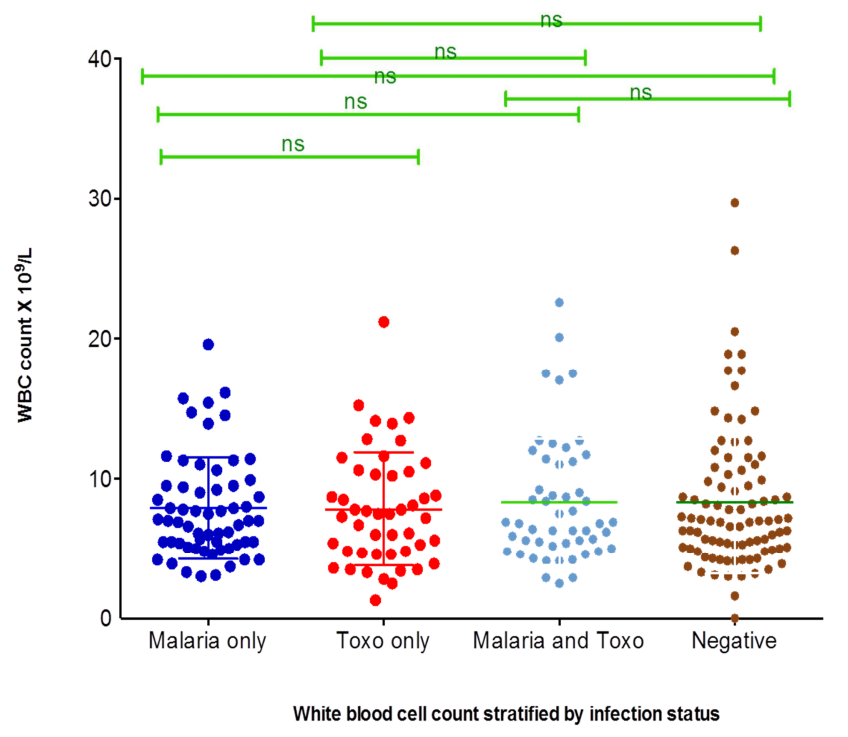

Figure 8. White blood cells distribution stratified by infection status.

\section{Platelets distribution stratified by infection status}

Analysis done to compare the various infection statuses and their effects on the platelets gave a statistical significance difference only between children who had toxoplasma infection and those who were negative for both infections $(\mathrm{P}=0.0139)$. The remaining comparisons were statistically insignificant with $\mathrm{p}$ values from top to bottom lines as follows $0.094 ; 0.510 ; 0.853 ; 0$ ).

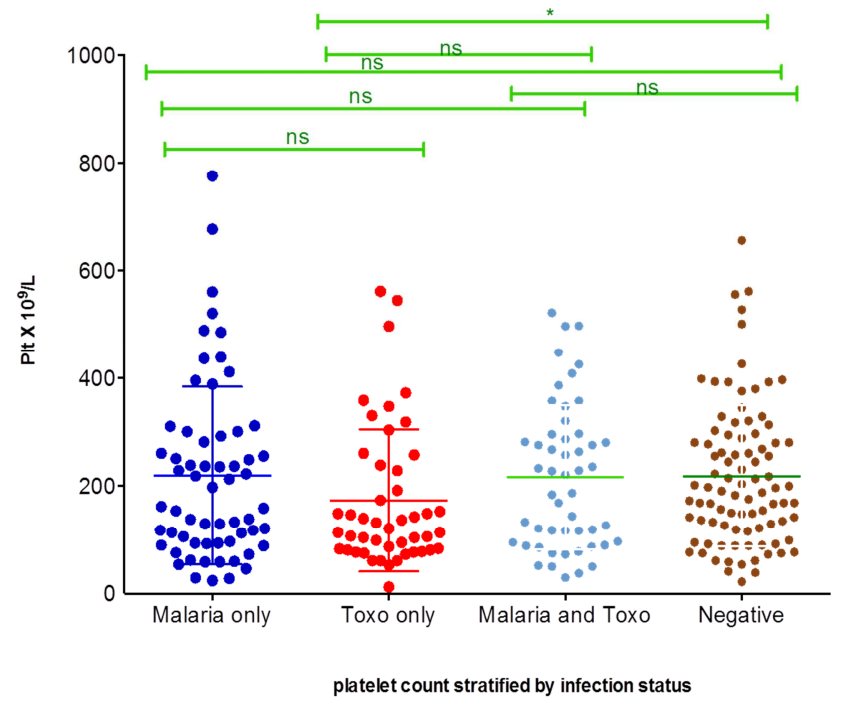

Figure 9. Platelet distribution stratified by infection status.

\section{Discussion}

Malaria remains a public health problem in Cameroon; the disease is responsible for $31 \%$ of consultations and $44 \%$ of 
hospitalizations in health facilities. It is responsible for $18 \%$ of deaths occurring in health facilities in the country. In children less than 5 years, $41 \%$ of deaths are due to malaria.[8] Between January and September of 2013, 182,402 cases of malaria were recorded in the Far North Region. The increase in the number of cases and deaths is observed every year between July and October, a period conducive for malaria transmission resulting from heavy rains and standing waters. Women and children are the most affected and child mortality in the region has increased (behind malaria).

Toxoplasmosis is becoming a global health hazard as it infects $30-50 \%$ of the world human population. The total prevalence rate of toxoplasmosis among children in Najaf/ Iraq was in a range of $48 \%$. [9] The dissemination of toxoplasmosis among children of both sexes has not been talked in detailed in Cameroon; most of the work was concentrated on the study of toxoplasmosis in pregnant women.

Toxoplasma gondii and plasmodium species are both endemic apicomplexan parasites that have been incriminated in the cause of febrile illnesses in children in the sub-Saharan regions of Africa. Moreover these parasites have some common routes of transmission, common receptors for pathogenicity and both effector of some hematological parameters. Despites this, little is known about the prevalence of Toxoplasmosis and malaria co-infection and their effects on hematological parameters and malaria parasite density reasons why this study aims at investigating the above in children (0-16 years) in the Nkolbisson health district.

A hospital based cross sectional study was adopted and well-structured questionnaires were administered to 315 children/guardians. The presence of toxoplasmosis and malaria co-infection was confirmed in 63 children giving an overall prevalence of $20 \%$. This percentage is considerable and in line with the fact that these parasites are both endemic in this region and may also be well explained due to the facts that both parasites can be transmitted vertically and can both causes of febrile illnesses in children. [10]

With respect to individual infection, the presence of the malaria parasite was confirmed in 135 children giving a prevalence of $42.8 \%$. This is almost similar to the $55.5 \%$ obtained by Eva, in Bipindi, Cameroon. [8] The highest prevalence of malaria was seen in males, who accounted for $168(53.5 \%)$ and the age group $0-5$ years $67(50 \%)$. In addition to the fact that males accounted for the majority of participants in this study and the modal age group being 0-5 years, it is mostly young children under the age of five in sub- Saharan Africa who bear the highest burden of this disease. Again, Eva in Bipindi, recorded a malaria prevalence of $53.09 \%$ among children a less than 5 years. [8]

This study showed a lower RBC count in majority of the malaria positive children $(57 \%)$. The cause and effect of malaria and anemia is complex and not fully understood. Infected RBCs display a reduced deformability and altered surface characteristics, which usually would lead to them being filtered and cleared by the spleen. However, $P$. falciparum has found a way to counter this protective measure. They modify their host cell membrane, which ultimately results to the cytoadherence of RBCs onto the endothelium. Infected and uninfected erythrocytes cluster together; a process called sequestration and rosetting, and clog up the capillary and post capillary venules of various organs. In addition, the enhanced destruction of uninfected erythrocytes coupled with a decrease in erythrocyte production all add to malaria related anemia. [11] Moreover, children between the ages of 0 to 5 years recorded high prevalence's of mild, moderate and severe anemia. This is partly due to the fact that this was the modal age group and they also had the greatest number of malaria infection. The least prevalence's for both malaria and anemia were noted in the older subjects 11-16 years.

On the other hand, $40 \%$ prevalence was obtained toxoplasmosis with $37 \%$ toxoplasma IgG and 3\% toxoplasma IgM. This prevalence of $40 \%$ is high as compared to the $26 \%$ obtained by Vincent, in Lagos, southern Nigeria, from November 2013 to March 2014. [12] The age group 0-5 years had the highest percentage of toxoplasmosis $(40 \%)$, which, is in line with the results of Jasim, in the Najaf province (Iraq), with a prevalence of $48 \%$ in this age group. [9] However the presence of $\mathrm{IgG}$ antibody in a majority of patients who participated in this research means that most of the infections were chronic.

Considering toxoplasmosis and malaria co-infection, various infection statuses were compared to see their effects on anemia, parasitemia and blood parameters. To beginning with the anemia stratification by infection statusFor comparison between those who had malaria only and toxoplasmosis only there was a one star (low) significance with $P$ value of $0.0374(p<0.05)$ where by those with malaria only had lower hemoglobin concentrations compared to those with toxoplasmosis only, this is contrary to the findings of Jasim, as he states that hemoglobin concentration indicates direct significant relationship with toxoplasmosis. [9] As for those who had malaria and those who were negative for both infection there was a one star significance with $p$ value of $0.0320(\mathrm{p}<0.05)$ this however explains the fact that anemia is a complication of malaria. With the other parameters there were no significance obtained but there were some considerable differences between the means of children with toxoplasmosis only and children with co-infection. However a lower HB concentration was expected in those with coinfection because both infections are thought to have anemia as a feature. [5]

Analysis done using the $\mathrm{T}$ test to compare the parasite density in those who had malaria only and children who had co-infection of malaria and toxoplasmosis, there was no statistically significance difference observed $(\mathrm{P}=0.1241)$. However potentially high parasitemia are due in part to the large number of merozoites produced and the ability of $\mathrm{P}$. falciparum to invade all erythrocytes, the parasitemia can also rapidly increase due to cytoadherence and sequestration of P. falciparum which eventually lead to most of the complications associated with $\mathrm{P}$. falciparum malaria as reported by Michelle in 2011. [1] Thus the presence of Toxoplasma gondii seems not to have an effect on malaria parasitemia.

On the part of white blood cells, there was no significant difference $(\mathrm{P}>0.05)$ between the WBC count of children who 
had malaria only, toxoplasmosis only, those who had a coinfection and those who had no infection at all. Manas showed thatleukocyte components were significantly affected during malaria infection Thailand children. [13] Neutrophil, lymphocyte, monocytes, eosinophil and basophil counts were all significantly decreased in patients with falciparum malaria and vivax malaria as compared to those with non-malaria group ( $\mathrm{P}$ value $<0.0001$ ). WBCs count is however may not be affected duringtoxoplasmosis. [10-13] It would have been expected that the total $\mathrm{WBC}$ count in malaria and toxoplasmosis co-infection to remain low but children infected with malaria and toxoplasmosis had a mean total WBC, which was higher than those with toxoplasmosis only. Could the presence of both parasites instead have a positive effect on the WBCs?

The trend in analysis done on platelets count were somehow similar to that observed in WBC where in children who had co-infection had higher mean platelet counts compared to those who had toxoplasmosis only and malaria only, whereas the presence of both would have been expected to have a negative effect on the platelet count. Manas obtained an $84.9 \%$ thrombocytopenia in the malaria-infected individuals. [13] In another study, the investigators noted that in congenital toxoplasmosis, six of the seven parasitologically proved cases examined had thrombocytopenia. [14] Consequently we expected to find low platelet count in malaria and toxoplasmosis co-infection but it was not the case. Probably, the presence of both infections could have a positive effect on platelet production. However, this needs to be confirmed by doing an in-vitro study using mice. Moreover, there was a statistically significance difference in platelet count in children who had toxoplasma infection and those who were negative for both toxoplasmosis and malaria $(\mathrm{P}=0.0139)$. However thrombocytopenia is a complication of toxoplasmosis. [14]

\section{Conclusion}

In this study, the prevalence of toxoplasmosis was $40 \%$, malaria $42.8 \%$ and toxoplasmosis and malaria co-infection $20 \%$. This study thus indicates a need to enforce control and preventive measures against these infections in Cameroonian children. The age group 0-5 years was identified as risk group for both infections and Nkol-Atem had the highest prevalence of both infections. Based on our results toxoplasmosis and malaria of co-infection led to an increase in RBCs, WBCs, and platelets counts in our study population. This could therefore be suggestive of a mechanism between the two parasites that may improve the physiology of blood cells production. Therefore the need for further investigation on the interactions of these two parasites remains imperative. However the presence of a co-infection did not show any influence on the malaria parasite density.

\section{Recommendations}

This study has a number of limitations as it cannot clearly explain the mechanisms that led to the observed effects of coinfection on hematological parameters. Additionally the data linkage to care may overestimate the proportion of children actually been treated for toxoplasmosis in Cameroon. Despite all these limitations this study provides valuable information on the prevalence of malaria and toxoplasmosis co-infection in children where data is almost unavailable more over it may also give grounds for further investigations in this domain. In view of the above limitations the following recommendations are made;

First and foremost, at the level of policy makers and other authorities, in order to achieve the national malaria and toxoplasmosis prevention and control plans, the capacity of diagnosing both malaria and toxoplasmosis should be strengthened by improving infant health care coverage, subsequent guardian's notifications, promoting retention in care and proper follow up of diagnosed persons.

Secondly, further prospective studies should be carried out to elucidate the dynamics between these two pathogens and how they affect hematological parameters.

This study goes further to recommend that health care professionals should strengthen their efforts in the diagnosis of febrile illnesses in children, particularly toxoplasmosis as cases may be missed due to improper diagnostic techniques.

Finally to the general population community and individual control measure should be followed to stop the route of transmission of these pathogens.

\section{Acknowledgement}

My profound gratitude goes to: The staffs and patients of the Nkolbisson health district Hospital, for allowing us to carry out this study, and for their participation and support; Thanks goes to UPCAWA SWEDEN for putting together the research team and their support.

\section{Authors' Contributions}

Samuel Nambile Cumber (Writing of the final manuscript/editing and supervised the abstract work from thesis form in to a summarized paper. Also attended to all the comments from the reviewers and made additional changes where necessary).

Kameni Nguekam Yvonne-Estelle (Collected Data and first draft of the manuscript)

\section{Appendix}

\section{Definition of terms and concepts}

- Toxoplasmosis

A common parasitic infection in humans, warm-blooded animals, and birds, transmitted by contact with the microorganism Toxoplasma gondii. The disease normally runs a mild course, with a few noticeable symptoms resembling those of the common cold. If contracted by a woman during pregnancy, however, toxoplasmosis can cause serious birth defects. In people whose immune system has been suppressed by age or other diseases, such as acquired immunodeficiency syndrome (AIDS), toxoplasmosis can cause severe nervous system disease (Microsoft Encarta, 2009).

\section{- Malaria}

A recurring illness caused by infection with single-celled 
parasites of the genus Plasmodium. Anopheles mosquitoes transmit these parasites from one person to another in their bites. Malaria is characterized by periodic bouts of severe chills and high fever. Serious cases of malaria can result in death if left untreated. More than a million people die of the disease each year, most of them in Africa, according to the World Health Organization (WHO). (Microsoft Encarta, 2009)

- Hematological parameters

This refers to the various components of blood (mainly the cell). A complete blood count (CBC), is a tests that indicates the number of red blood cells, white blood cells, and platelets in a given unit of blood, their values infer different physiological states. Changes in hematological parameters are likely to be influenced by any disease condition including endemic diseases. [8]

\section{References}

[1] Michelle L. Tonkin. Science, July 2011 "Host cell invasion by Apicomplexan parasites": Insights from the structure of AMA1 in complex with a RON2 peptide dx.doi.org/10.1126/science. 1204988 .

[2] Center for disease control, 2015 Global Health - Division of Parasitic Diseases and Malaria. Lastly reviewed march 2015.

[3] Montoya JG, 2008. Laboratory diagnosis of Toxoplasma gondii infection and toxoplasmosis. J Infect Dis C; 185 (Suppl 1): $\mathrm{S} 73-82$.

[4] Moulin F, Lesage F, Legros AH, Maroga C, Moussavou A, Guyon P, Marc E, Gendrel D. 2003 Thrombocytopenia and Plasmodium falciparum malaria in children with different exposures. Arch Dis Child.; 88: 540-541. doi: 10.1136/adc.88.6.540.

[5] Al-Mousawi, G. N. (2008): Some Immunological and seroepidemiological aspects associated with toxoplasmosis in aborted and unmarried women in Basrah province. M. Sc. Thesis, University of Basrah.
[6] Di Carlo P, Romano A, Schimmenti MG, Mazzola A, Titone L. 2008 Materno- fetal Toxoplasma gondii infection: critical review of available diagnostic methods. Infez Med; 16 (1): $28-32$.

[7] Nissapatorn V, Azmi Noor MA, Cho SM, Fong MY, Init I, Rohela M. (2003) Toxoplasmosis; Prevalence and risk factors. J ObstetGynaecol; 23: 618-624.

[8] Eva Songue, Claudel Tagne, Pretty Mbouyap, Paul Essomba, Roger Moyou -Somo 2013. Epidemiology of Malaria in three Geo-Ecological Zones along the Chad-Cameroon Pipeline.

[9] Jasim Hameed Taher, 2009 "Seroepidemiological aspects of toxoplasmosis among pre-school children in Najaf Province" Technical Institute $\backslash$ Kufa - Iraq.

[10] Fayer R, Dubey JP, Lindsay DS: Zoonotic protozoa: from land to sea. Trends Parasitol 2004, 20: 531-536.

[11] Haldar K, Mohandas N. Malaria, erythrocytic infection, and anemia. Hematology Am SocHematolEduc Program. 2009: 87-93. doi: 10.1182/asheducation-2009.1.87 Eva Songue Plan stratégique national de luttecontre le paludisme 2011-2015. Ministère de la Santé Publique. Cameroun. 2012: 82p.

[12] Vincent Pam Gyang, OlaoluwaPheabianAkinwale, Yueh-Lun Lee, Ting-Wu Chuang, AkwaowoOrok, OlusolaAjibaye, Chien-Wei Liao, Po-Ching Cheng, Chia-Mei Chou, YingChieh Huang, Kuo-Hua Fan, Chia-Kwung Fan 2014 "Toxoplasma gondii infection: seroprevalence and associated risk factors among primary schoolchildren in Lagos City, Southern Nigeria" http://dx.doi.org/10.1590/0037-8682-03102014.

[13] Manas Kotepui, Bhukdee Phunphuech, Nuoil Phiwklam, Chaowanee Chupeerach, and Suwit Duangmano, 2014 "Effect of malarial infection on haematological parameters in population near Thailand-Myanmar border" Published online 2014 Jun 5. doi: 10.1186/1475-2875-13-218.

[14] Passos ST, Silver JS, O'Hara AC, Sehy D, Stumhofer JS, Hunter CA. IL-6 promotes NK cell production of IL- 17 during toxoplasmosis. J Immunol; 2010, 184: 1776-83. 